\title{
Framework for Contextualized Learning Ecosystems
}

\author{
Mario Muñoz-Organero ${ }^{1}$, Gustavo A. Ramírez², Pedro J. Muñoz-Merino ${ }^{1}$ \\ and Carlos Delgado Kloos ${ }^{1}$ \\ ${ }^{1}$ Universidad Carlos III de Madrid \\ Av. Universidad 30 E-29811 Leganés Madrid (Spain) \\ \{munozm, pedmume, cdk\} @it.uc3m.es \\ ${ }^{2}$ Universidad del Cauca Popayán Colombia \\ gusramir@gmail.com
}

\begin{abstract}
Using mobile personal devices to interact with pervasive smart learning objects and services that create contextualized learning ecosystems can enhance both the learning outcomes and the motivational states of students. This paper defines and analyzes several pervasive learning ecosystems in which students at the Carlos III University of Madrid interact with contextualized learning objects and services. The technology defining the contextualized learning environments is first introduced and later used in two user experiments. These experiments provide data both about the learning outcomes for students after interacting with smart learning objects and services and about the motivational impact that the use of these technologies have on them.
\end{abstract}

Keywords: Learning ecosystems, contextualized learning in an Internet of Things, Learning outcomes, student motivation enhancement, user experiments, RFID, NFC, mobile learning.

\section{Introduction}

Ubiquitous computing embeds computational capabilities into contextualized objects creating an invisible part of the fabric of everyday life [1]. Providing communication and computational capabilities to smart objects adds a new dimension to an any-time any-where and for any-body pervasive computing environment into an Internet of Things designed to communicate to and from any-thing. "The Internet of Things will enable forms of collaboration and communication between people and things, and between things themselves, hitherto unknown and unimagined" [2]. Human users can make use of mobile personal devices to interact with such an Internet of things [3]. Depending on the purpose of the user when interacting with an Internet of Things, several ecosystems can be defined [4]. Learning ecosystems in an Internet of Things enable anyone to learn at anytime and anywhere [1] by interacting with contextualized smart objects.

The use of mobile devices in learning activities as an interaction mechanism with contextualized pervasive learning ecosystems can enhance the shift from pure instructor centered classroom teaching to constructivist learner centered educational settings [5] in which students interact with smart learning objects and services in a 
contextualized environment. Several experiments during the last years have concentrated on studying how the use of mobile phones and PDAs in educational settings can enhance the learning process and learning outcomes. A context-aware language-learning support system using PDAs, GPS and RFID tags is described in [6]. The paper in [7] proposes a context-aware ubiquitous English learning system in a campus environment which can detect the learning location by wireless positioning techniques and retrieve specific learning content based on location information to individual learners through wireless networks. An environment for learning with educational resources based on RFID and ubiquitous computing technologies is described in [8]. A similar experiment which uses a context-aware ubiquitous learning environment which utilizes mobile devices, sensors and wireless networks to conduct situated learning is described in [9].

Among the main characteristics normally present in experiments using mobile devices in learning settings three of them are of particular importance for the scope of this paper: their use for exploratory learning, in situated environments, for problem solving. In exploratory learning, the students investigate a system on their own, often in pursuit of a goal. Exploratory learning [10] is a constructivist instructional approach, wherein the learners are encouraged to explore and to experiment on their own. Situated learning was defined by Brown et al. as "embedded within and inseparable from participating in a system of activity deeply determined by a particular physical and cultural setting" [11]. Problem based learning (PBL) originates form education in medicine [12] and consists of the manipulation of problematic situations, comprising the appraisal of the problem, creation of a problem space, the selection of goals and the deployment and monitoring of cognitive structures to reach those goals [13].

Among the main objectives targeted when using mobile devices in learning experiments two of them are of especial importance for the scope of this paper: improving the learning outcomes and enhancing the motivation of students. Liu et al. [8] evaluated the impact of using mobile devices in a situated learning environment on the grades of the students. Moura and Carvalho [14] studied if the use of mobile phones and podcasts encouraged the students to learn. The activity theory was used in [15] for designing ubiquitous learning scenarios showing that learners become motivated and engage in active pursuit and construction of knowledge when using ubiquitous technologies.

This paper defines and analyzes several pervasive learning ecosystems in which students at the Carlos III University of Madrid interact with contextualized learning objects and services. The technology defining the contextualized learning environments is first introduced and later used in two user experiments. These experiments provide data both about the learning outcomes for students after interacting with smart learning objects and services and about the motivational impact that the use of these technologies have on them.

\section{Defining Contextualized Pervasive Learning Ecosystems}

The vision of the Internet of Things extends the connectivity to the Internet into our everyday lives through the use of wireless technologies and identification 
mechanisms such as RFID which generate technology enhanced pervasive ecosystems [4]. This section presents an analysis and construction proposal for a particular case of such ecosystems: the contextualized pervasive ecosystems for learning.

The definition of the concept of the contextualized learning ecosystem should incorporate together the use of three technology enhanced components: pervasive learning objects, pervasive learning services and proactive learning environments. Combining together technology enablers such as RFID tags, mobile devices and pervasive service items, learning environments can be created in which users interact with pieces of knowledge embedded in communicating smart objects and contextualized services. Using appropriate technologies such as NFC (Near Field Communications) [16] integrated in user devices such as smart-phones, the users can consume the information provided by pervasive (either passive or active) learning objects. The process in which the user consumes this ambient knowledge can be interactive either by using the communication link with the pervasive learning object or by using an external representation of such object in the Internet [17]. The second component of a contextualized learning ecosystem, the learning service, provides additional learning capabilities such as projection rooms, printing services or collaborative information sharing to the learning environment. The assimilation of concepts and the acquisition of practical competencies can be simplified by the use of such services. Finally, the third component in a pervasive learning ecosystem should also allow accidental learning to take place in a user friendly manner. Proactive learning environments should be used to provide smart, contextualized and user adapted learning information when the user needs it without requiring the user intervention.

This section presents some technological tools developed inside the Gradient group at the Carlos III University of Madrid to define a contextualized pervasive learning ecosystem categorized as pervasive learning objects, pervasive learning services and proactive learning environments.

\subsection{Pervasive Learning Objects}

Pervasive learning objects can be either physical things and tools (such as the different cards in a telecommunication exchange or a working engine inside a car) or conceptual representations of them (such as a panel or a poster displaying how to send a packet over an IP network). Pervasive learning objects in a contextualized situated environment should provide multimedia information to nearby users by means of a wireless communication link such as NFC or Bluetooth (remote access can also be provided to such objects over the Internet such as in remote labs [18]). Figure 1a captures a user interacting with a telecommunication exchange using an NFC enabled mobile phone (Nokia 6131 NFC). Multimedia information is obtained from NFC tags in different parts of the exchange either directly or by following a link to an external object in the Internet (which can be cached inside the mobile device for efficiency). Figure 1b captures a virtual recreation of a physical environment using an NFC enhanced panel presenting information about how to configure certain services in an IP network. A user can interact with the panel using an NFC compatible mobile device. 

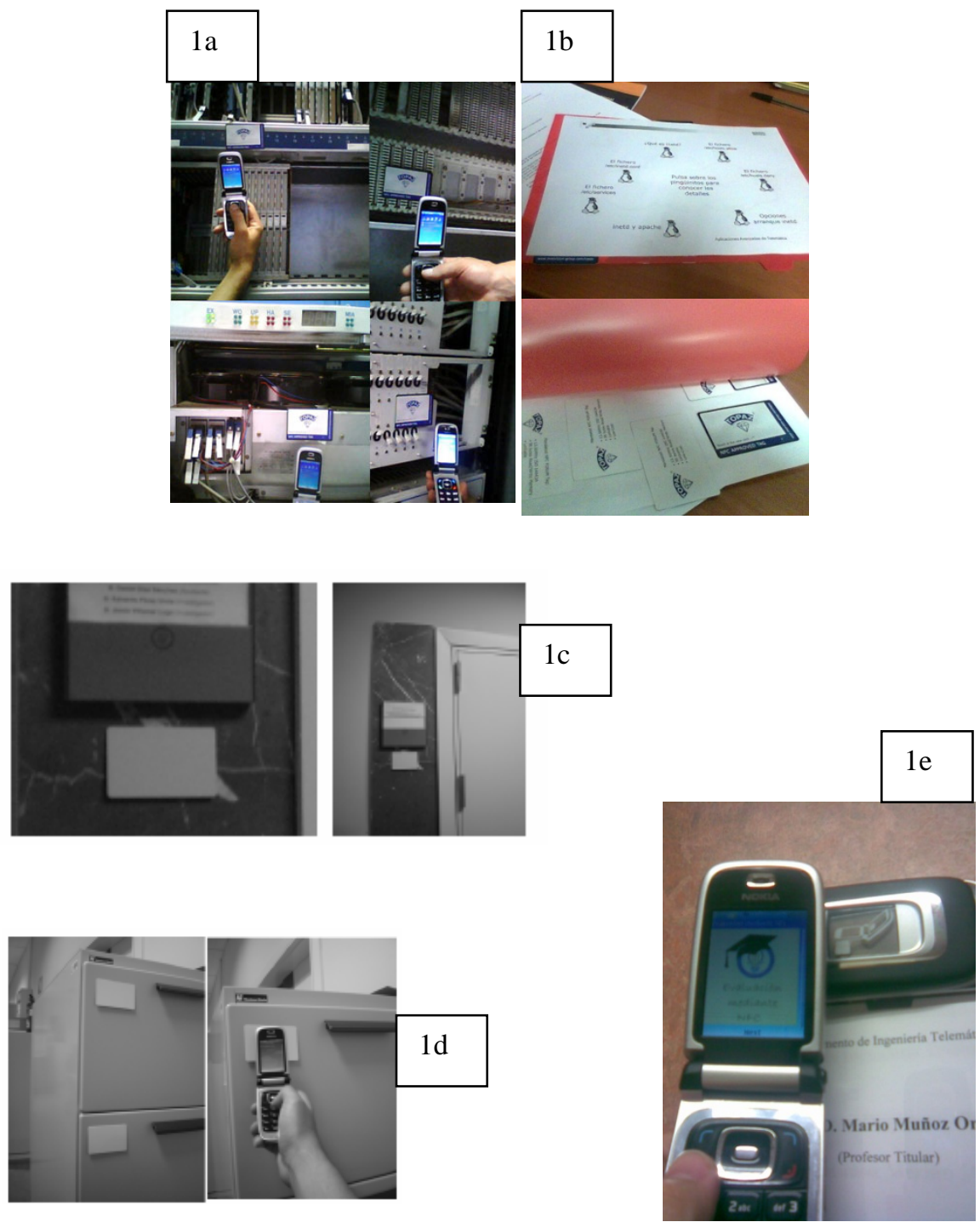

Fig. 1. Pervasive learning objects. 1a shows a user interacting with NFC tags in a telecommunication exchange. $1 \mathrm{~b}$ shows the front and back of a panel containing NFC tags to interact with a mobile user. 1c presents a touching note associated to an office. 1d shows a touching cabinet and 1e presents a bidirectional learning object using HTTP over NFC.

Any existing object in a learning environment can be tagged with information. Figures $1 \mathrm{c}$ and 1d capture two examples more [19]. Figure 1c represents a touching note associated to any office or lecture room in a university. A student can touch the note to get information about the location of a particular professor or about the activity been carried out in a particular lecture room at a particular time. The touching 
note also allows the student to leave a message back to the professor if required. Figure 1d captures a touching cabinet in which information is provided about the contents of such objects or about who has interacted with them.

A contextualized learning ecosystem can also incorporate active learning objects augmented with bidirectional input/output facilities. Students should be able not only consume the contents of smart learning objects but also to interact with them. Several technologies can be applied for implementing embedded interactions in an Internet of Things [20]. Figure 1e captures the interaction of an NFC enabled mobile phone running our implementation of an HTTP browser [21] accessing the information on an HTTP server over a bidirectional NFC channel. Our implementation of the HTTP server [22] is executed in a similar NFC enabled mobile device providing contextualized access to students touching on it. Figure 1e implements an examination platform deployed at the door of the office of one of the authors of this paper. The student receives a short, single choice type exam that after completion can be uploaded to the examination platform (the NFC enabled phone at the door) receiving instant feedback about the answers.

\subsection{Pervasive Learning Services}

A second family of components in contextualized pervasive learning ecosystems is constituted by pervasive learning services offered to nearby learners. Services embedded in smart pieces of the contextualized learning environment should provide instant access to learning facilitators such as printers, projection panels or interactive platforms for collaborative editing and document processing. Figure 2a captures one of such services: a projection panel controlled using an NFC mobile device [23]. The back part of the projection panel contains an array of NFC tags that are detected by the mobile phone and sent to the PC controlling the projection of the information. The reading of a tag in the panel by the mobile device is mapped to a click of a mouse at the appropriate location.
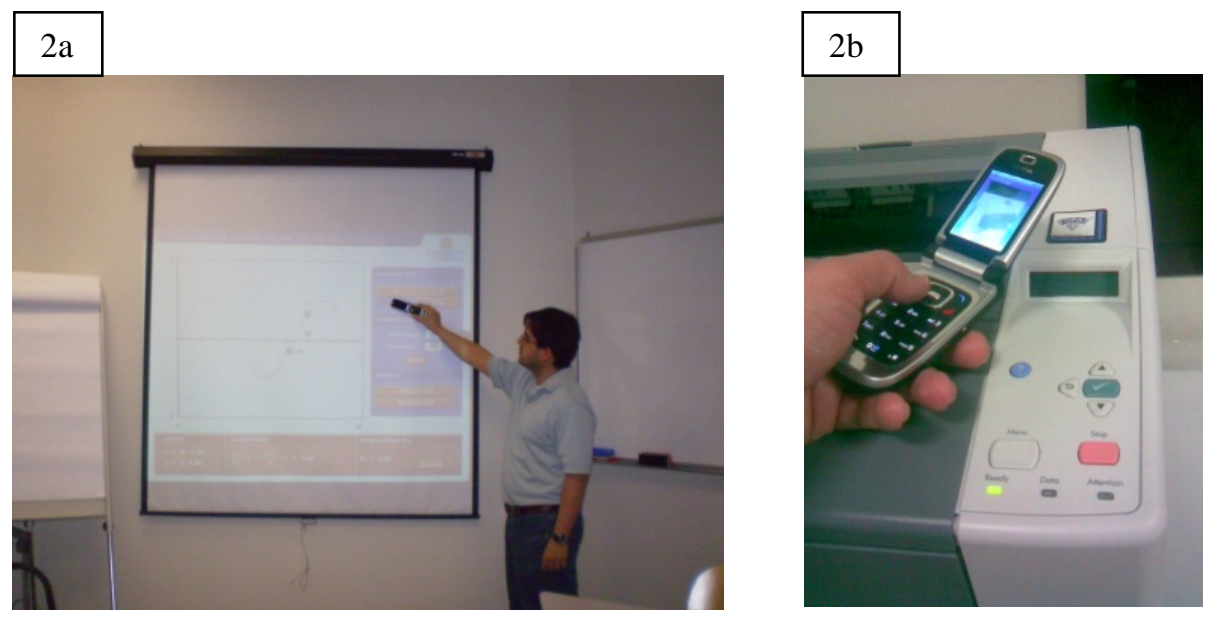

Fig. 2. Pervasive learning services. 1a presents a projection panel controlled using an NFC mobile device. $2 b$ shows an NFC enabled printing service. 
Figure $2 \mathrm{~b}$ captures our implementation of a printing service. A student can print an image, a presentation of slides or a pdf document simply by selecting it on his or her mobile phone and by touching an NFC tag on the printer with it. This tag contains the Bluetooth address of a controlling computer to which to send the resource to be printed. The controlling computing opens that resource with a reading application and sends it to printing queue of the printer. A similar interaction interface has also been implemented for controlling the image projected in the panel in figure $2 \mathrm{a}$ so that a learner can share and discuss information with other nearby students.

Other examples of services that we have implemented for the creation of contextualized pervasive learning ecosystems are a document sharing application controlling a PC hosted document using NFC and Bluetooth enabled mobile devices and a "touchable beamer" projecting an image simply by touching it with an NFC mobile device.

\subsection{Proactive Learning Environments}

Contextualized pervasive learning ecosystems should facilitate and enhance both deliberate and accidental learning. Pervasive learning objects and services described in previous subsections are mainly targeted to a learning user having the control of the learning interaction process in a deliberate manner (the learning user is normally responsible for starting the interaction process). Proactive learning environments, on the other hand, should be used to provide smart, contextualized and user adapted learning information when the user needs it without requiring the user intervention in an accidental manner. Proactive parts in a pervasive learning ecosystem should know what the learning user needs by inferring what the user is, has and does and provide contextualized and relevant information to this user. [20] presents some examples of proactive environments in an Internet of Things such as a kitchen. We have concentrated on the creation of proactive learning ecosystems. This section describes two examples.

A first example of a proactive learning environment that we have implemented is a smart gate at the entrance of the Torres Quevedo building inside the Carlos III University of Madrid campus in Leganes (Madrid, Spain). A laptop with the profile of some students (the group of the experiment) uses Bluetooth to detect such students when they go inside that building. Depending on the subjects that each student is taking the system sends relevant information to that student using OBEX object transfer over bluetooth.

A second example is a proactive application for sending learning pills to interested students in class (later described as a case study in this paper). As in the previous example, OBEX object transfer over Bluetooth is used to automatically send information to students. In this case, the information sent is a set of complementary exercises to reinforce what is being taught by the professor at this particular moment in class. 


\section{Case Studies}

This section captures two case studies that we have carried out in order to assess both the learning outcomes for students after interacting with smart learning objects and services and the motivational impact that the use of pervasive learning technologies have on them.

\subsection{Interactive Panels}

An interactive panel such as the one presented in Figure $1 \mathrm{~b}$ has been used to simulate a server room running some network services in Linux. Students attending the "advanced telematic applications" course at the Carlos III University of Madrid (dedicated to teach third year Telecommunication Engineering students how to configure IP network services such as DNS, NFS, NIS, SMTP and HTTP) were divided into control and experiment groups. The group of the experiment used an NFC panel such as the one in figure $1 \mathrm{~b}$ while the control group attended a traditional face-to-face instructor-led class. Our objective was to show if using an NFC panel alone a student is able to obtain a similar learning gain as in a traditional face-to-face instructor-led class (but with no limitations in when, where and how fast to learn). In order to have enough data for statistical analysis, two different experiments were carried out.

In the first experiment, the 31 students attending the class were randomly divided into two groups, one of 10 students (the group of the experiment) and the other of 21 students (the control group). The control group received a normal lecture about the main characteristics of some services in a TCP/IP network. The session took place in a classroom with slides showing a diagram containing multiple servers as a simulated servers' room and was explained by the professor of the course. The group of the experiment was moved to another room with NFC mobile phones. Students interacted with NFC panels as presented in Figure $1 \mathrm{~b}$ simulating the interaction with the physical servers in the servers' room. The learning objects used were based on video and audio and contained the same information that the professor gave to the control group in class. The students were able to control the learning experience by exploring the panels at their own pace, replaying the contents if needed. No synchronous communication channel was provided to allow students to ask questions to an online instructor.

At the beginning of the session, each student of both groups answered a pre-test with questions about the contents of the experiment. The objective of this pre-test was to measure any prior knowledge that students may have had so that the learning gain could be measured at the end of the experiment. The test consisted of 7 questions that were rated on a scale between 0 and 7 . Once the session finished, both groups were asked to answer a post-test containing again 7 questions to assess the learning gain of the process in both groups.

In the second experiment, the 24 students attending the class were again randomly divided into two groups, one of 10 students (the group of the experiment) and the other of 14 students (the control group) taking into account that students in the group of the experiment in the first experiment were not again in the group of the experiment in this second experiment. The methodology was the same. The learning 
content described the details of the "inetd" service, its configuration files in a Linux system and its component architecture.

The learning gains for the two groups in the two experiments are captured in Table 1 (represented by the difference between pre-test and post-test). The learning gains for the group of the experiment were slightly worse in the first experiment but slightly better in the second one.

In order to assess if the learning gains of the group of control are similar to those of the group of the experiment or not an Anova test was performed to the learning gains for the 4 samples of students (including the two groups in the two experiments). The $F$ value obtained is 1.37 which is smaller than the critical value 2.79 and the p-value obtained is 0.26 which is bigger than the critical value of 0.05 . Therefore it can be assumed that the differences in the learning gains are due to random factors.

Table 1. Average increase of results by experiment

\begin{tabular}{lll}
\hline Group & First experiment & Second experiment \\
\hline Group of Experiment & 1.9 & 3.22 \\
Control Group & 2.43 & 2.93 \\
\hline
\end{tabular}

Some intrinsic motivation related factors were also evaluated in these experiments using a questionnaire at the end of them. The questions answered by students were (using a Likert-type scale):

- How would you define your intrinsic motivation towards the use of mobile devices in class? (1. Very small, 2. Small, 3 Neither small nor big, 4. Big, 5. Very big)

- Comparing learning with mobile devices and traditional instructor based classes, what do you enjoy most? (Learning with mobile devices: 1. Much less, 2. Less, 3 The same, 4. More, 5. Much more)

- Would you be willing to do a second experiment like this in the future? (1. Strongly disagree, 2. Disagree, 3 Neither agree or disagree, 4. Agree, 5. Strongly Agree)

Table 2 captures the answers of students. Students recognized that they were motivated by the use of mobile devices in class. Similar results are presented in [14] for the use of mobile phones and podcasts. The use of mobile devices can be used therefore to increase the motivational state of students. Students also reported that they slightly prefer learning with mobile devices instead of learning in a traditional environment. Finally, students also said that they would enjoy doing a similar experiment a second time.

Table 2. Answer of the students to motivation questions

\begin{tabular}{lllllll}
\hline Questions & 1 & 2 & 3 & 4 & 5 & Mean \\
\hline 1 & 0 & 0.08 & 0.34 & 0.5 & 0.08 & 3.58 \\
2 & 0 & 0.17 & 0.58 & 0.25 & 0 & 3.08 \\
3 & 0 & 0.08 & 0.17 & 0.5 & 0.25 & 3.92 \\
\hline
\end{tabular}




\subsection{Proactive Learning Pills}

A second case study has been deployed and evaluated implementing a proactive application for sending learning pills to interested students in class as described in section 2.3. A set of complementary exercises was sent to the mobile phones of 38 students via Bluetooth to reinforce what was taught by the professor in class. A total of 6 learning pills were sent in 6 different classes to students attending the "advanced telematic applications" course at the Carlos III University of Madrid (using a different group of students than in the previous case study). As in the previous case study, both learning gains and motivational impacts have been assessed.

In order to validate if the introduction of proactive learning pills in class promotes an increase in learning gains of students of the course (in a scale from 0 to 10), the group of 60 students was divided in a control group (those students with no o little use of the learning pills) and the group of the experiment (those students consuming at least half of the pills). The learning gains were assessed using a pre-test at the beginning of the course and a post-test at the end. The results are captured in table 3 . The results clearly show that students regularly consuming the learning pills obtained a bigger increase in their learning outcomes. This increase is also influenced by other factors that were also present in the experiment such as the fact that students receiving the pills were also physically attending the lectures where many of the students that did not receive the pills were not.

Table 3. learning gains for students consuming at least half of the pills and the rest of students

\begin{tabular}{ll}
\hline Group & Learning gain \\
\hline Group of Experiment & 5.4 \\
Control Group & 1.6 \\
\hline
\end{tabular}

The motivational impact that the use of the learning pills had on students was assessed using a questionnaire. The answers of the students to the question: "Does the use of mobile phones for receiving learning pills increase your motivation for the subject?" using a Likert-type scale (1. Not at all, 2. Very Slightly, 3. Somehow, 4. Significantly, 5. Very much) is captured in table 4 . The results are divided into two groups: students consuming at least half of the pills (frequent users) and the rest of the students (sporadic users). Although there is a bigger impact on students frequently consuming the pills, event sporadic users of the pills considered more than significant the motivational impact that the learning pills had on them.

Table 4. motivational impact for students consuming at least half of the pills and the rest of students

\begin{tabular}{ll}
\hline Group & Motivational impact \\
\hline Frequent users & 4.18 \\
Sporadic users & 4.07 \\
\hline
\end{tabular}




\section{Conclusions}

This paper has studied and analyzed the basic components of pervasive contextualized learning ecosystems defining and implementing some learning objects, services and proactive environments. The paper has also presented how the use of pervasive learning smart objects and services can create contextualized learning ecosystems that enhance both learning outcomes and motivational states of students interacting with them using mobile personal devices. This paper has presented the results of two case studies that implemented pervasive learning ecosystems in which students at the Carlos III University of Madrid interacted with contextualized learning objects and services. These experiments have provided data both about the learning outcomes for students after interacting with smart learning objects and services and about the motivational impact that the use of these technologies have on them.

Acknowledgements. The research leading to these results has been partially funded by the ARTEMISA project TIN2009-14378-C02-02 within the Spanish "Plan Nacional de I+D+I", the Madrid regional community projects S2009/TIC-1650 and CCG10-UC3M/TIC-4992 and the SOLITE CYTED Program 508AC0341. Thanks to INNOVISION for providing the NFC tags for this experiment. Gustavo RamirezGonzalez is funded by the EU Programme Alban, scholarship number E06D101768CO and by the Universidad del Cauca.

\section{References}

1. Sakamura, K., Koshizuka, N.: Ubiquitous computing technologies for ubiquitous learning. In: IEEE International Workshop on Wireless and Mobile Technologies in Education, WMTE 2005, November 28-30, pp. 11-20 (2005)

2. International Telecommunication Union ITU, ITU Internet Reports 2005: The Internet of Things (2005)

3. Wiechert, T., Thiesse, F., Michahelles, F., Schmitt, P., Fleisch, E.: Connecting Mobile Phones to the Internet of Things: A Discussion of Compatibility Issues between EPC and NFC. In: Americas Conference on Information Systems (AMCIS), Keystone, Colorado, USA (2007)

4. Welbourne, E., Battle, L., Cole, G., Gould, K., Rector, K., Raymer, S., Balazinska, M., Borriello, G.: Building the Internet of Things Using RFID: The RFID Ecosystem Experience. IEEE Internet Computing 13(3), 48-55 (2009)

5. Holzinger, A., Nischelwitzer, A., Meisenberger, M.: Mobile Phones as a Challenge for mLearning: Examples for Mobile Interactive Learning Objects (MILOs). In: IEEE International Conference on Pervasive Computing and Communications Workshops, Third IEEE International Conference on Pervasive Computing and Communications Workshops, Hawai, pp. 307-311 (2005)

6. Ogata, H.: Computer Supported Ubiquitous Learning: Augmenting Learning Experiences in the Real World. In: Fifth IEEE International Conference on Wireless, Mobile, and Ubiquitous Technology in Education (wmute 2008), Beijing, China, pp. 3-10 (2008)

7. Hsieh, H.C., Chen, C.M., Hong, C.M.: Context-Aware Ubiquitous English Learning in a Campus Environment. In: 7th IEEE International Conference on Advanced Learning Technologies (ICALT 2007), Niigata, Japan, pp. 351-353 (2007) 
8. Liu, T.Y., Chu, Y.L., Tan, T.H., Chang, C.C.: RFID-based Ubiquitous Learning Environment for Outdoor Learning. In: 7th IEEE International Conference on Advanced Learning Technologies (ICALT 2007), Niigata, Japan, pp. 675-677 (2007)

9. Wu, T.T., Yang, T.C., Hwang, G.J., Chu, H.C.: Conducting Situated Learning in a Context-Aware Ubiquitous Learning Environment. In: Fifth IEEE International Conference on Wireless, Mobile, and Ubiquitous Technology in Education, Beijing, China, pp. 82-85 (2008)

10. Bruner, J.S.: The Act of Discovery. Harvard Educational Review 31, 21-32 (1961)

11. Brown, J.S., Collins, A., Duguid: Situated Cognition and the Culture of Learning. Educational Researcher 18(1), 32-42 (1989)

12. Barrows, H.S., Tamblyn, R.M.: An evaluation of problem-based learning in small groups utilizing a simulated patient. Journal of Medical Education 51, 52-54 (1976)

13. Newell, A., Simon, H.A.: Human Problem Solving. Prentice-Hall, Englewood Cliffs (1972)

14. Moura, A., Carvalho, A.A.: Mobile Learning: Teaching and Learning with Mobile Phones and Podcasts. In: Eighth IEEE International Conference on Advanced Learning Technologies, Santander, Spain, pp. 631-633 (2008)

15. An, J.: Activity Theory for Designing Ubiquitous Learning Scenarios. In: Innovative Techniques in Instruction Technology, E-learning, E-assessment, and Education, pp. 205-209. Springer, Netherlands (2008)

16. Ortiz Jr., S.: Is near-field communication close to success? IEEE Computer Publication, Los Alamitos (2006)

17. Yan, L., Zhang, Y., Yang, L.T., Ning, H., Zhang, Y.: The Internet of Things From RFID to the Next-Generation Pervasive Networked Systems. Auerbach Publication (March 2008)

18. Garcia-Zubia, J., Lopez-de-Ipia, D., Ordua, P.: Mobile Devices and Remote Labs in Engineering Education. In: Eighth IEEE International Conference on Advanced Learning Technologies, ICALT 2008, pp. 620-622 (2008)

19. Gonzalez, G.R., Organero, M.M., Kloos, C.D.: Early Infrastructure of an Internet of Things in Spaces for Learning. In: Eighth IEEE International Conference on Advanced Learning Technologies, ICALT 2008, July 1-5, pp. 381-383 (2008)

20. Kranz, M., Schmidt, A., Holleis, P.: Embedded Interaction - Interacting with the Internet of Things. In: IEEE Internet Computing Online (December 2009) (accepted for future publication), doi:10.1109/MIC.2009.141

21. Muñoz-Organero, M., Muñoz-Merino, P.J., Delgado Kloos, C.: Enabling M-Learning Applications In MIDP Devices: Specification And Development Of An HTML To MIDP Automatic Converter. In: International Technology, Education and Development Conference, Valencia (March 2007)

22. Muñoz-Organero, M., Delgado Kloos, C.: Web-enabled middleware for mobile devices. In: International Wireless Applications and Computing 2007 Conference, Lisbon, Portugal, July 6-8 (2007)

23. Ramírez, G., Organero, M.M., Kloos, C.D., Astaiza, Á.C.: Exploring NFC Interactive Panel. In: The Fifth Annual International Conference on Mobile and Ubiquitous Systems: Computing, Networking and Services (MobiQuitous 2008), Dublin, Ireland, July 21-25 (2008) 